\title{
Las actitudes de los jóvenes internados en la prisión de Córdoba ante los procesos de reeducación
}

\section{The attitudes of the young persons in the prison of Cordoba. Towars the reeducational function}

\author{
María Milagrosa luque Jiménez \\ lamiluji@hotmail.com \\ María del Rosario Ruiz-Olivares ${ }^{1}$ \\ M92rorum@uco.es \\ Universidad de Córdoba, España
}

\section{Resumen:}

La educación y la integración social son derechos de todas las personas, incluso de las que están en prisión, por lo que las instituciones penitenciarias deben esforzarse por realizar una labor reeducadora y de reinserción. La actitud que mantengan estas personas hacia la reeducación puede ser importante en la implicación hacia dicho proceso educativo. El objetivo de este trabajo de investigación es conocer las actitudes de los internos de la prisión de Córdoba (España), menores de veinticinco años, hacia la reeducación, e intentar indagar qué variables están asociadas a dichas actitudes. Este trabajo se ha realizado con un diseño de investigación descriptivo ex post facto transversal y con una muestra de 50 internos menores de veinticinco años de la prisión de Córdoba (España). A partir de los resultados parece que más de la mitad de los internos tienen una actitud positiva hacia la función reeducadora del centro penitenciario. A modo de conclu-

\begin{abstract}
:
Educational and social integrations are universal rights, and even those people who are in prison should have them. So penal institutions must strive to rehabilitative and re-educate inmates. These people's attitudes to re-education might be important in the involvement towards such process. Thus, the objective of this piece of research is to know the attitudes of inmates, under 25, at the prison of Cordoba (Spain) towards re-education, and to try to find out which variables are associated with attitudes. This study has been done with a descriptive research design ex post facto cross and with a sample of 50 inmates under the age of twenty-five in the prison of Córdoba (Spain). From the results, it seems that more than half of the inmates have a positive attitude towards the rehabilitative function of the penal institution. In conclusion, a high attitude toward re-education can benefit not only the re-
\end{abstract}

1 Dirección para correspondencia (Correspondence address):

María Del Rosario Ruiz-Olivares. Facultad de Ciencias de la Educación. Universidad de Córdoba. Avd. San Alberto Magno, s/n. 14071 Córdoba (España). 
Las actitudes de los jóvenes internados en la prisión de Córdoba ante los procesos de reeducación

María Milagrosa luque Jiménez y María del Rosario Ruiz-Olivares

sión, una actitud alta hacia la reeducación beneficia no sólo la reeducación del interno, sino también su reinserción social.

\section{Palabras clave:}

Actitud; reeducación; jóvenes; prisión. habilitation of the inmate, but also their social reintegration.

\section{Key words:}

Attitude; prison; re-education; youth.

\section{Résumé:}

Toute personne a le droit à l'éducation et à l'intégration sociale, même celles qui sont en prison, ce pour quoi les institutions pénitentiaires doivent s'efforcer pour réaliser un travail rééducateur et de reinsertion. L'attitude que ces personnes maintiennent envers la rééducation peut être importante dans lıimplication vers le dit processus éducatif. L`objectif de ce travail de recherche est de connaître les attitudes des internes de la prison de Cordoue (Espagne), mineurs de vingt-cinq ans, envers la rééducation, et diessayer de savoir quelles variables sont associées aux dites attitudes. Ce travail a été réalisé avec une méthodologie de recherche descriptive ex-post facto transversal et avec un échantiIlon de 50 internes âgés de moins de vingt-cinq ans de la prison de Cordoue (Espagne). À partir des résultats, il semble que plus de la moitié des internes ont une attitude positive envers la fonction rééducatrice du centre pénitentiaire. En matière de conclusion, une haute attitude envers la reéducation peut bénéficier non seulement à la rééducation de l)interne, mais aussi à sa réinsertion sociale.

\section{Mots clés:}

Une attitude; une reeducation; des jeunes; une prison.

Fecha de recepción: 6-9-2013

Fecha de aceptación: 28-3-2014

\section{Introducción}

A nivel coloquial, las instituciones penitenciarias han sido tradicionalmente consideradas como lugares de encierro, castigo y aislamiento social y no como un espacio en el que desarrollar procesos de reeducación y reinserción social, tal y como se indica en la legislación española (Martín, 2009). Teniendo en cuenta el artículo 25.2 de la Constitución Española queda constancia que el cumplimiento de las penas privativas de libertad estará orientado hacia la reeducación y la reinserción social. Por lo tanto, los centros penitenciarios deben cumplir funciones educativas, reeducadoras y de reinserción social. Según Martín (2006), estos procesos de reeducación y de reinserción social se desarrollan dentro de un marco institucional concreto con una serie de condicionantes, como la importancia que se le da a los aspectos de orden, seguridad y disciplina, en contra posición a las posibles intervenciones educativas; su falta de permeabilidad con el exterior; el nivel cultural y educativo precario 
de sus usuarios; las escasas ofertas educativas y socioculturales; la poca estabilidad de la población en el mismo centro; el déficit de recursos económicos, técnicos y humanos; el bajo nivel socioeconómico de los internos; la evidente falta de libertad; etc. Todo esto dificulta en gran medida la puesta en marcha de acciones reeducadoras. A estos condicionantes propios de la institución y de la población penitenciaria, hay que añadirle otras variables que también tienen cierta influencia en dichas acciones. Martin (2006) las agrupa en variables ambientales orientadas a la vigilancia y no a lo educativo; variables regimentales, como por ejemplo, los horarios, las visitas, los traslados, las sanciones, etc.; variables psicosociales, como el consumo de drogas, problemas familiares, cambios en el estado de ánimo, etc.; y variables personales. Aspectos que pueden dificultar en gran medida la acción reeducadora.

Dentro del centro penitenciario, y siguiendo el esquema de la única investigación precedente hasta el momento (Martín, 2006), se ha optado por indagar en la población más joven del centro penitenciario de Córdoba, por varias razones: una de ellas es porque se considera que la etapa joven es la etapa más activa, por lo tanto, el riesgo de recaída es mayor (Kaiser, 1988); otra razón, es porque son parte de la futura población que probablemente va a nutrir el sistema penitenciario en los años futuros (Beristain, 1985); y por último, porque a estas edades la labor reeducadora tiene mayores posibilidades de provocar efectos positivos en la persona.

Con todo esto, indagar en la actitud de los internos más jóvenes hacia la función reeducadora parece interesante ya que puede orientar en la planificación de medidas educativas más eficaces. Por actitud se entiende aquella predisposición comportamental adquirida hacia un objeto o situación dada. Esta predisposición comportamental, está relacionada con tres componentes (Bolívar, 2002): por un lado, el afectivo, relacionado con el sentir; el cognitivo, relacionado con el saber; y por otro lado, el conativo, relacionado con el actuar, consistiendo estos tres componentes en evaluaciones que realizamos las personas ante cualquier situación. Así, los sentimientos pueden ser positivos o negativos, las percepciones favorables o desfavorables, y la conducta de apoyo u hostil (Martín, 2006). De igual modo, como indican Morales y Moya (2007), la actitud no es algo directamente observable, sino que es una variable latente, que refleja una evaluación global positiva o negativa de la actitud, es decir, refleja la tendencia favorable o desfavorable de una 
Las actitudes de los jóvenes internados en la prisión de Córdoba ante los procesos de reeducación

María Milagrosa luque Jiménez y María del Rosario Ruiz-Olivares

persona hacia una situación concreta, y es previa a la realización de cualquier comportamiento, favoreciendo el comportamiento si es positiva y evitándolo si es negativa. También es importante delimitar qué se entiende por reeducación. Como el término sugiere, consiste en educar o enseñar nuevamente algo. Garrido y Montoro (1.992) concibe la reeducación como el conjunto de actividades de trabajo social, psicológico, educativo, cultural y de formación profesional que se pueden programar, realizar y evaluar en todos o en la mayoría de los establecimientos penitenciarios. Concretamente, en las Reglas Penitenciarias Europeas, se entiende por reeducación penitenciaria: "cualquier medida tomada para conservar o recuperar la salud física o mental de los internos, facilitar su reinserción social y mejorar las condiciones generales de su reclusión". Pero para que la función reeducadora y la reinserción social tengan un resultado positivo parece necesario que las personas a las que va dirigida dicha intervención tengan una actitud positiva adecuada, teniendo en cuenta, como se ha comentado anteriormente, que las actitudes nos predisponen a realizar respuestas de tipo afectivo, cognitivo y comportamental (Bolívar, 2002).

Con todo esto, cuando se habla de la intervención en el medio penitenciario es necesario hacer referencia a dos áreas de trabajo: el área de régimen, que se ocupa de cuestiones burocráticas y de seguridad; y el área de tratamiento, que se ocupa de la reinserción y reeducación del interno. En esta última área es dónde se centra este trabajo de investigación, entendiendo el tratamiento como "el trabajo en equipo de especialistas, ejercido individualmente sobre el delincuente, con el fin de anular o modificar los factores negativos de su personalidad y dotarle de una formación idónea, para así apartarse de la reincidencia y lograr su readaptación a la vida social" (Martín, 2006, 140). Nuestro actual sistema penitenciario contempla una serie de actividades para la reeducación: actividades que comprenden todos los niveles de formación (Primaria, Secundaria, Bachiller, Formación Profesional Ocupacional, etc.), actividades socioculturales y deportivas, y trabajo penitenciario.

Con todo esto, esta investigación tiene el objetivo principal de conocer las actitudes de los jóvenes menores de veinticinco años internados en el Centro Penitenciario de Córdoba (España), ante la función reeducadora de la Institución Penitenciaria, y en cualquier caso, indagar qué variables están asociadas a las actitudes, ya que conociéndolas, se po- 
drán diseñar programas de intervención más adecuados que mejoren la predisposición de los internos para reinsertarse en la sociedad.

\section{Método}

\section{Participantes}

Para llevar a cabo la investigación nos hemos centrado en los internos jóvenes del Centro Penitenciario de Córdoba, concretamente los jóvenes menores de veinticinco años, basándonos en los criterios ya comentados en la introducción (Martín, 2006).

La población penitenciaria menor de veinticinco años en el Centro Penitenciario de Córdoba (España) en el momento de realizar la investigación era de 57 internos, aceptando participar en la investigación 50 internos. De estos 50 sujetos, 45 son hombres y 5 son mujeres. Son españoles el 76\%, estableciéndose la edad media en 22,78.

\section{Instrumentos}

Para realizar la investigación se han utilizado los siguientes instrumentos:

Un cuestionario de variables socioeducativas (Martín, 2006): como la edad, el sexo, los estudios, la experiencia laboral; los datos familiares, como estudios de los padres, situación laboral de los padres, antecedentes familiares en prisión; datos relativos al mundo delincuencial, como consumo de drogas, participación en delitos, influencias recibidas para delinquir, necesidad o no de cometer delitos; datos penitenciarios, como situación penal y penitenciaria, actividad realizada dentro del centro penitenciario y ocupación del tiempo de ocio.

También se utilizó otro cuestionario de actitudes hacia la reeducación, C.A.R. (Martín, 2006): Este cuestionario presenta un formato de respuesta tipo Likert, en el que los jóvenes tienen que posicionarse "muy de acuerdo", "de acuerdo", "indiferente", "en desacuerdo", "muy en desacuerdo", en un total de 18 ítems agrupados en torno a cuatro áreas de contenidos:

- Área I. Actitud que los internos tienen ante la función reeducadora del medio penitenciario.

- Área II. Actitud que los internos tienen ante las actividades que se realizan en el medio penitenciario. 
Las actitudes de los jóvenes internados en la prisión de Córdoba ante los procesos de reeducación

María Milagrosa luque Jiménez y María del Rosario Ruiz-Olivares

- Área III. Actitud que los internos tienen respecto de sí mismo ante la función reeducadora del medio penitenciario.

- Área IV. Actitud que los internos tienen sobre el grado en el que esperan reinsertarse en el futuro gracias a la labor del medio penitenciario.

\section{Diseño}

El objetivo de la investigación se ha valorado a través de un diseño descriptivo transversal donde se ha valorado la actitud hacia la reeducación de los internos de la prisión de Córdoba con el cuestionario de Actitudes hacia la Reeducación (C.A.R.) (Martín, 2006). Dicha puntuación se ha relacionado con las variables socioeducativas y sociodemográficas como: el nivel de estudios anterior al ingreso en prisión, nivel actual de estudios, consumo de drogas anterior al ingreso en prisión, consumo actual de drogas, situación penitenciaria, situación penal, sentirse valorado y recompensado por la actividad que realiza en prisión, antecedentes familiares en prisión, nivel de estudios de los padres, tiempo transcurrido en prisión continuada, ingreso en Centros de Menores, trato recibido de la familia, apoyo familiar en prisión, tipología delictiva, violencia al cometer el delito, influencias para delinquir, motivos por los que cometió delitos y actividad que realiza en prisión.

Se consideraron variables independientes las siguientes, agrupadas en cuatro ámbitos de estudio:

- Nivel de estudios: Nivel de estudios anterior al ingreso en prisión; Nivel actual de estudios; Recuerdo de la edad escolar.

- Relaciones familiares: Antecedentes familiares en prisión; Trato recibido de la familia; Apoyo familiar en prisión; Nivel de estudios del padre; Nivel de estudios de la madre.

- Contacto con la marginalidad: Ingreso en Centros de Menores; Tipología delictiva; Violencia al cometer el delito; Consumo de drogas anterior al ingreso en prisión; Consumo actual de drogas.

- Vida en prisión: Situación penitenciaria; Situación penal; Tiempo transcurrido en prisión continuada; Actividad que realiza en prisión; Trabajo que realiza en prisión; Sentirse valorado y recompensado por la actividad que realiza en prisión; Influencias para delinquir; Motivos por los que cometió delitos.

Y como variable dependiente:

- La variable dicotómica actitud baja/alta hacia la reeducación valorada con el cuestionario anteriormente descrito C.A.R. 


\section{Procedimiento}

Los cuestionarios fueron aplicados en el Centro Penitenciario de Córdoba (España). Se reunieron a los internos menores de veinticinco años en la clase de cada módulo, dónde se les explicó brevemente el objetivo de la investigación, y qué es lo que tenían que hacer para cumplimentar correctamente y de forma individual el cuestionario que se les entregaba. También se les informó que la contestación a los cuestionarios era voluntaria y anónima, accediendo prácticamente todos a colaborar.

\section{Análisis de datos}

Concluida la etapa de recogida de datos, abordamos el análisis de los mismos con el programa estadístico SPSS.

\section{Resultados}

Teniendo en cuenta que el objetivo de esta investigación era conocer las actitudes de los jóvenes menores de veinticinco años internados en el Centro Penitenciario de Córdoba (España), ante la función reeducadora de la Institución Penitenciaria, y en cualquier caso, e indagar qué variables están asociadas a la actitud, lo primero que se hizo fue dicotomizar la variable actitud de los jóvenes internados en la prisión de Córdoba ante la función reeducadora del medio penitenciario y así formar dos grupos de usuarios, unos con actitud alta y otros con actitud baja. Teniendo en cuenta la puntuación media $(54,22)$, se agruparon los sujetos en dos grupos, quedando el $48 \%$ (24) con actitud baja y el 52\% (26) con actitud alta.

Una vez dicotomizada la variable dependiente y teniendo en cuenta las variables independientes, se realizó un análisis de correlación con la prueba de independencia Chi cuadrado. 
Las actitudes de los jóvenes internados en la prisión de Córdoba ante los procesos de reeducación

María Milagrosa luque Jiménez y María del Rosario Ruiz-Olivares

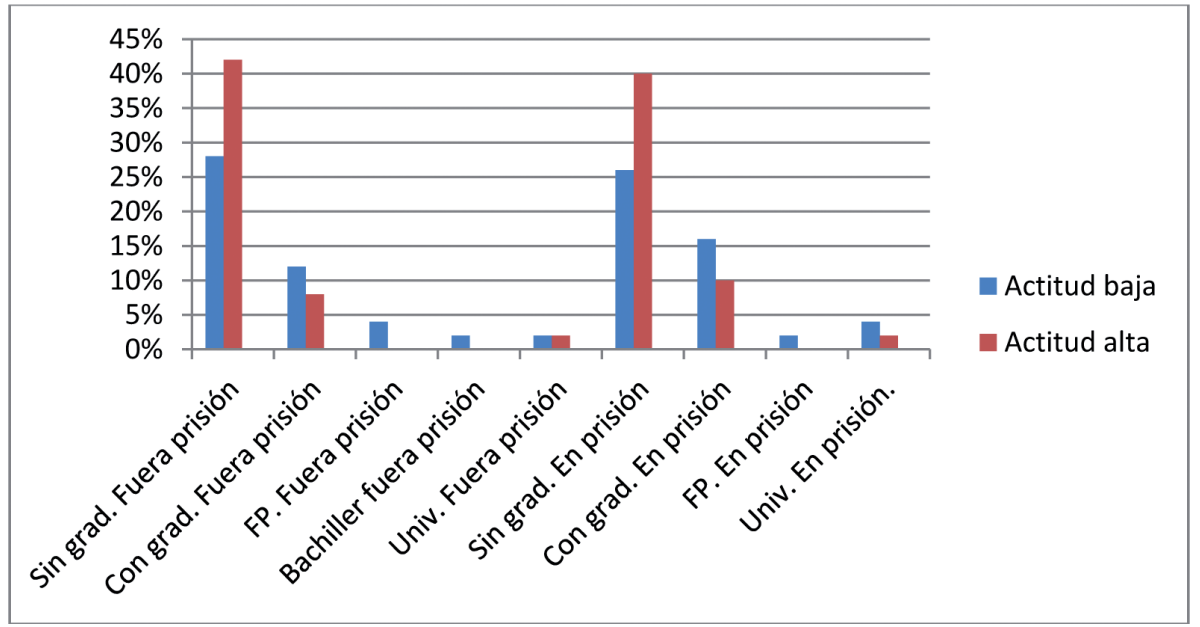

Figura 1. Nivel de estudios al ingresar en prisión y mejora del nivel de estudios en prisión

Parece que los sujetos que ingresan en prisión sin graduado tienen mejor actitud que los sujetos que ingresan con algún tipo de estudio, sin embargo, la relación entre el nivel de estudios al ingresar en prisión y la actitud mantenida hacia la reeducación es estadísticamente no significativa $\left(\chi^{2}=, 316 ; p>0,05\right)$. Asimismo, los sujetos que continúan sin graduado dentro de prisión tienen mejor actitud que los sujetos con algún tipo de estudio. Sin embargo, la relación entre la mejora del nivel de estudios producida en prisión y la actitud mantenida hacia la reeducación es estadísticamente no significativa $\left(\chi^{2}=, 329 ; p>0,05\right)$.

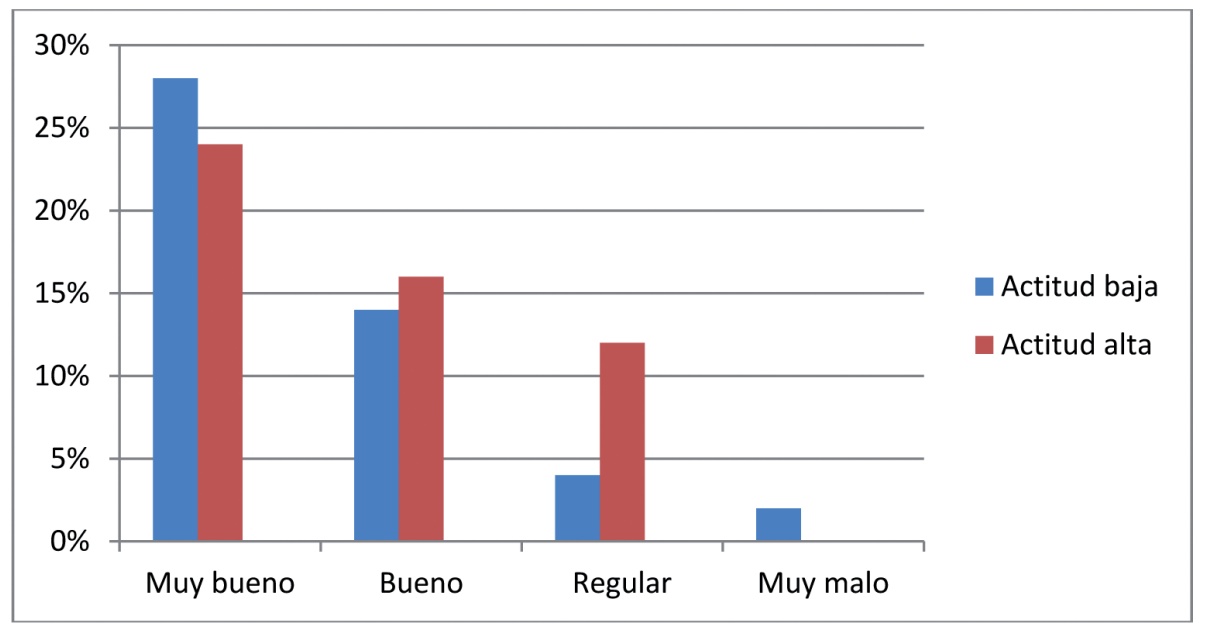

Figura 2. Recuerdo de la infancia escolar 
Parece que los sujetos que tienen un recuerdo "bueno" y "regular" tienen mejor actitud que los sujetos que tienen un recuerdo "muy bueno", sin embargo, la relación entre el recuerdo de la infancia escolar y la actitud mantenida hacia la reeducación es estadísticamente no significativa $\left(\chi^{2}=, 370 ; p>0,05\right)$.

Respecto el ámbito, nivel de estudios de los internos, el nivel de estudios al ingresar en prisión, la mejora del nivel de estudios en prisión y el recuerdo de la infancia escolar no propician una actitud alta hacia la reeducación.

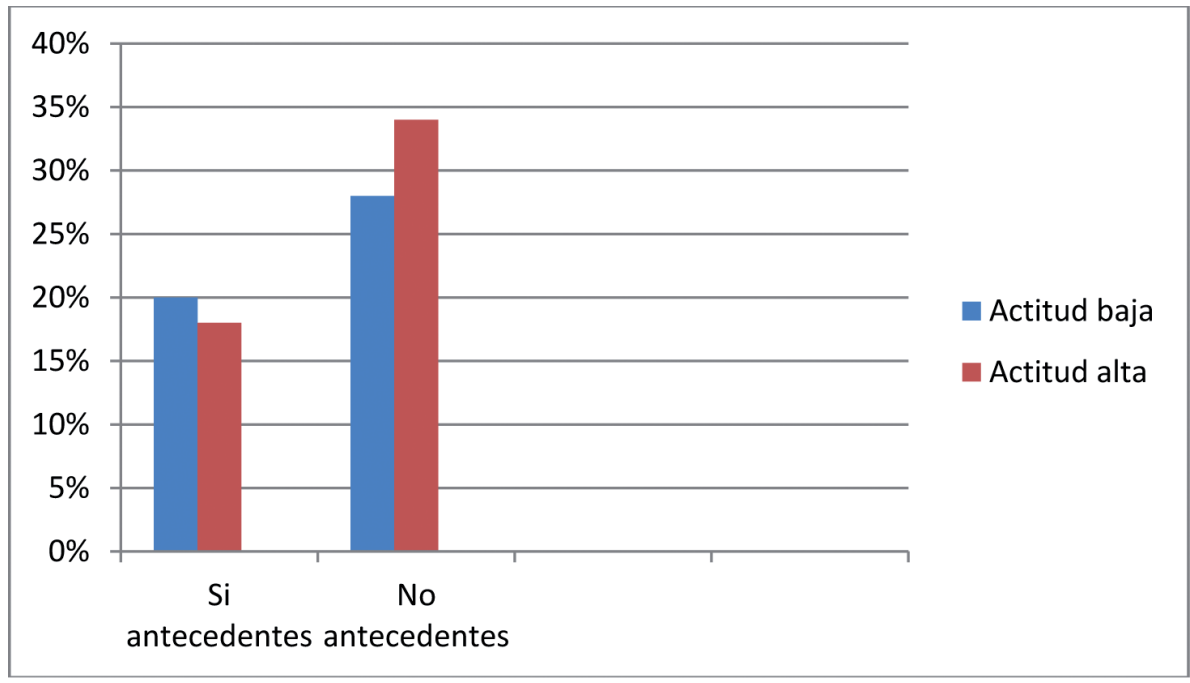

Figura 3. Antecedentes familiares en prisión

Parece que los sujetos que no tienen antecedentes familiares en prisión tienen mejor actitud que los sujetos que si tienen, sin embargo, la relación entre los antecedentes familiares en prisión y la actitud mantenida hacia la reeducación es estadísticamente no significativa $\left(\chi^{2}=, 608\right.$; $p>0,05)$. 
Las actitudes de los jóvenes internados en la prisión de Córdoba ante los procesos de reeducación

María Milagrosa luque Jiménez y María del Rosario Ruiz-Olivares

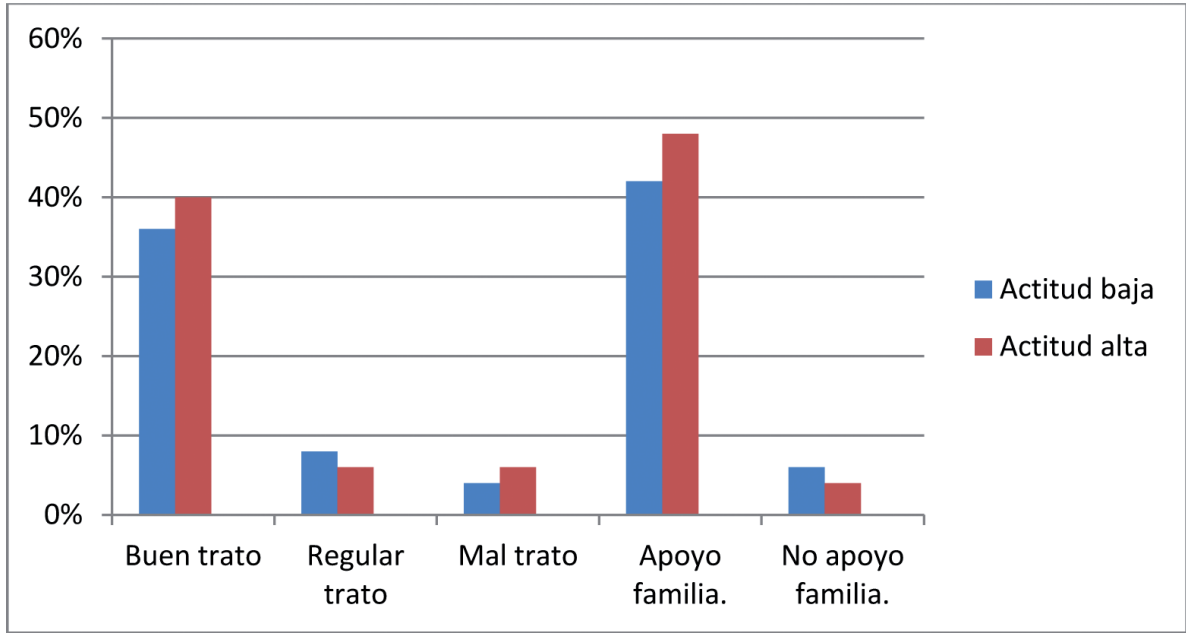

Figura 4. Trato recibido de la familia y apoyo familiar

Parece que los sujetos que han recibido un buen trato familiar tienen mejor actitud que los sujetos que no han recibido buen trato, sin embargo, la relación entre el trato recibido de la familia y la actitud mantenida hacia la reeducación es estadísticamente no significativa $\left(\chi^{2}=, 832 ; p\right.$ $>0,05)$. Asimismo, los sujetos que reciben apoyo familiar tienen mejor actitud que los sujetos que no reciben apoyo de su familia, sin embargo, la relación entre recibir apoyo familiar y la actitud mantenida hacia la reeducación es estadísticamente no significativa

$$
\left(\chi^{2}=, 571 ; p>0,05\right) \text {. }
$$

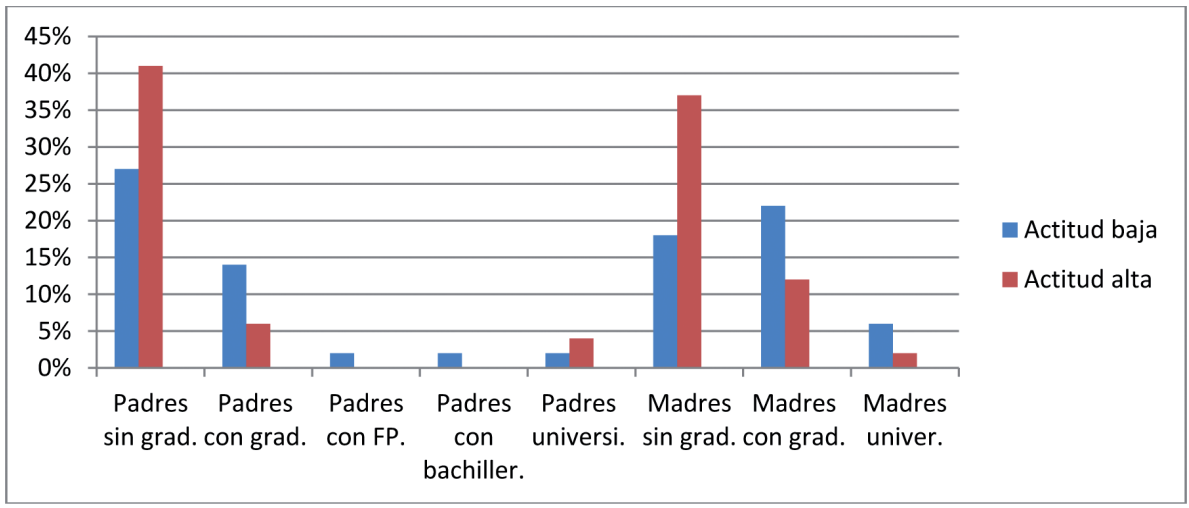

Figura 5. Nivel de estudio de los padres 
Parece que los sujetos cuyo padre no tiene graduado escolar tienen mejor actitud que los sujetos cuyo padre si tiene graduado, sin embargo, la relación entre el nivel de estudios del padre y la actitud mantenida hacia la reeducación es estadísticamente no significativa $\left(\chi^{2}=, 254 ; p\right.$ $>0,05)$. Asimismo, los sujetos cuya madre no tiene graduado escolar tienen mejor actitud que los sujetos cuya madre si tiene graduado, sin embargo, la relación entre el nivel de estudios de la madre y la actitud mantenida hacia la reeducación es estadísticamente no significativa $\left(\chi^{2}\right.$ $=, 067 ; p>0,05)$.

En resumen, en el ámbito relaciones familiares, muestran actitud alta hacia la reeducación los sujetos que no tienen antecedentes familiares en prisión, han sido bien tratados por la familia, reciben apoyo familiar y los que tienen padres y madres sin graduado escolar.

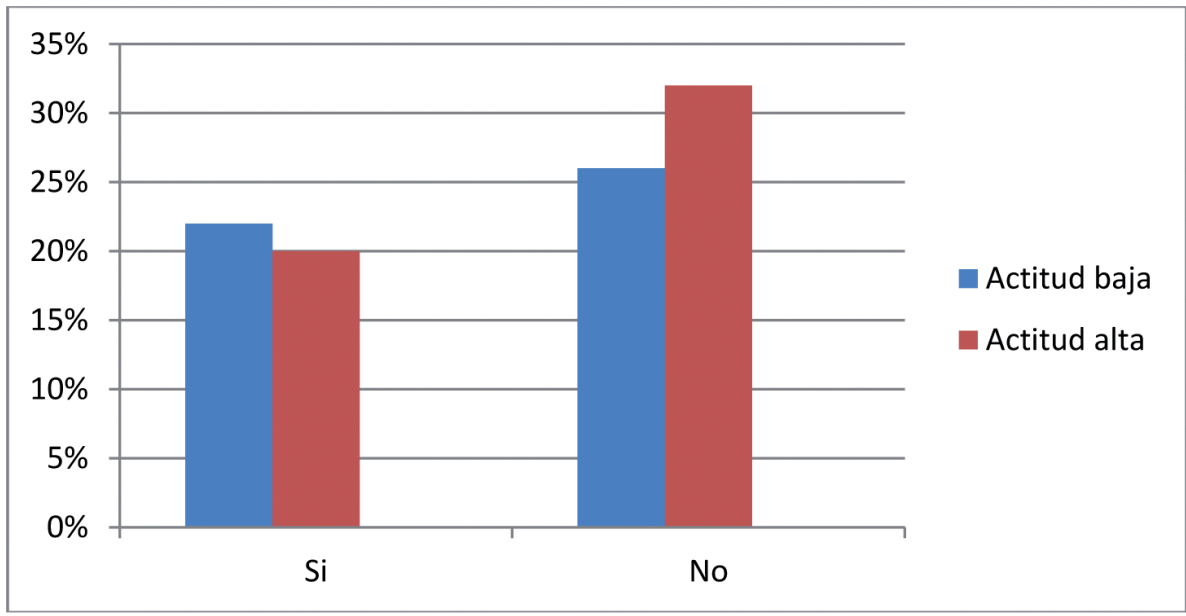

Figura 6. Haber estado ingresado en centros de menores

Parece que los sujetos que no han estado ingresados en centros de menores tienen mejor actitud que los sujetos que han estado ingresados, sin embargo la relación entre haber estado ingresado en centro de menores y la actitud mantenida hacia la reeducación es estadísticamente no significativa $\left(\chi^{2}=, 598 ; p>0,05\right)$ 
Las actitudes de los jóvenes internados en la prisión de Córdoba ante los procesos de reeducación

María Milagrosa luque Jiménez y María del Rosario Ruiz-Olivares

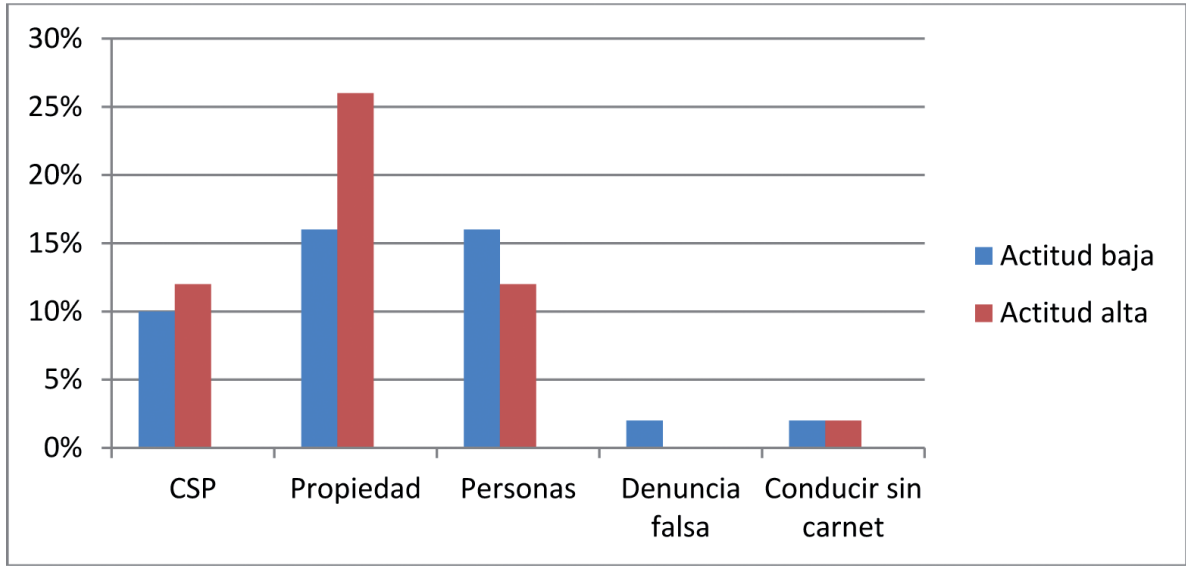

Figura 7. Tipología delictiva

Parece que los sujetos que han cometido delitos contra la salud pública o contra la propiedad tienen mejor actitud que los sujetos que han cometido delitos contra personas, sin embargo, la relación entre el tipo de delito y la actitud mantenida hacia la reeducación es estadísticamente no significativa $\left(\chi^{2}=, 664 ; p>0,05\right)$

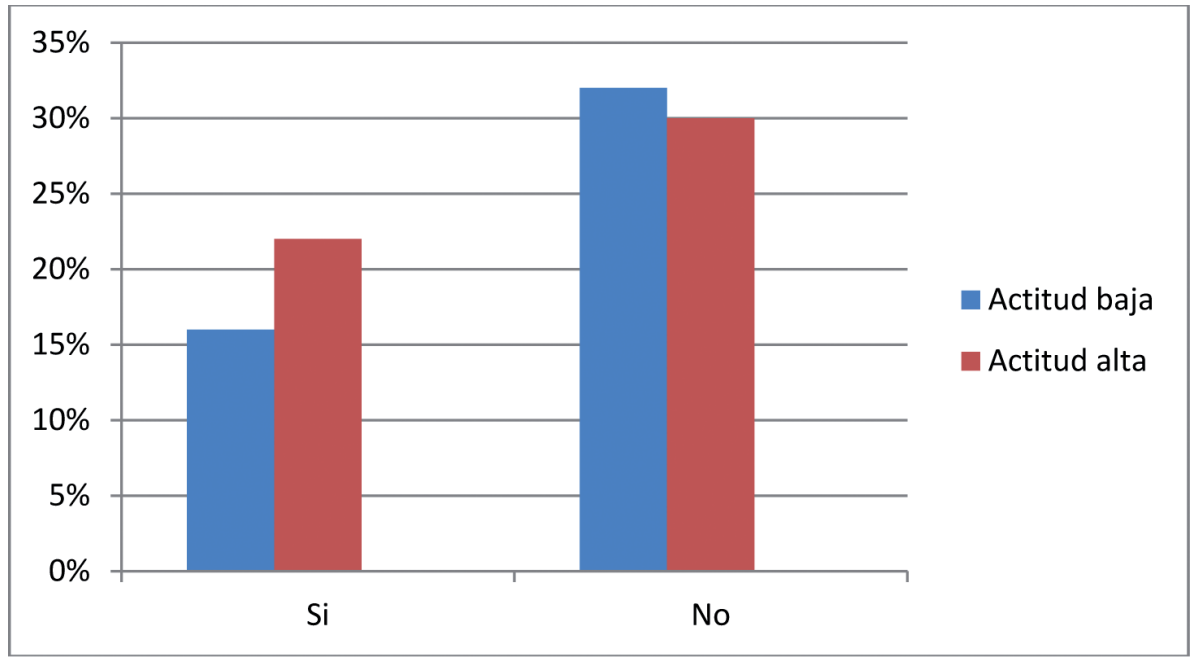

Figura 8. Uso de violencia en el acto delictivo

Parece que los sujetos que usaron violencia en el acto delictivo tienen mejor actitud que los sujetos que no usaron violencia, sin embargo, la relación entre el uso de violencia en el acto delictivo y la actitud mantenida hacia la reeducación es estadísticamente no significativa $\left(\chi^{2}=, 514 ; p>0,05\right)$. 


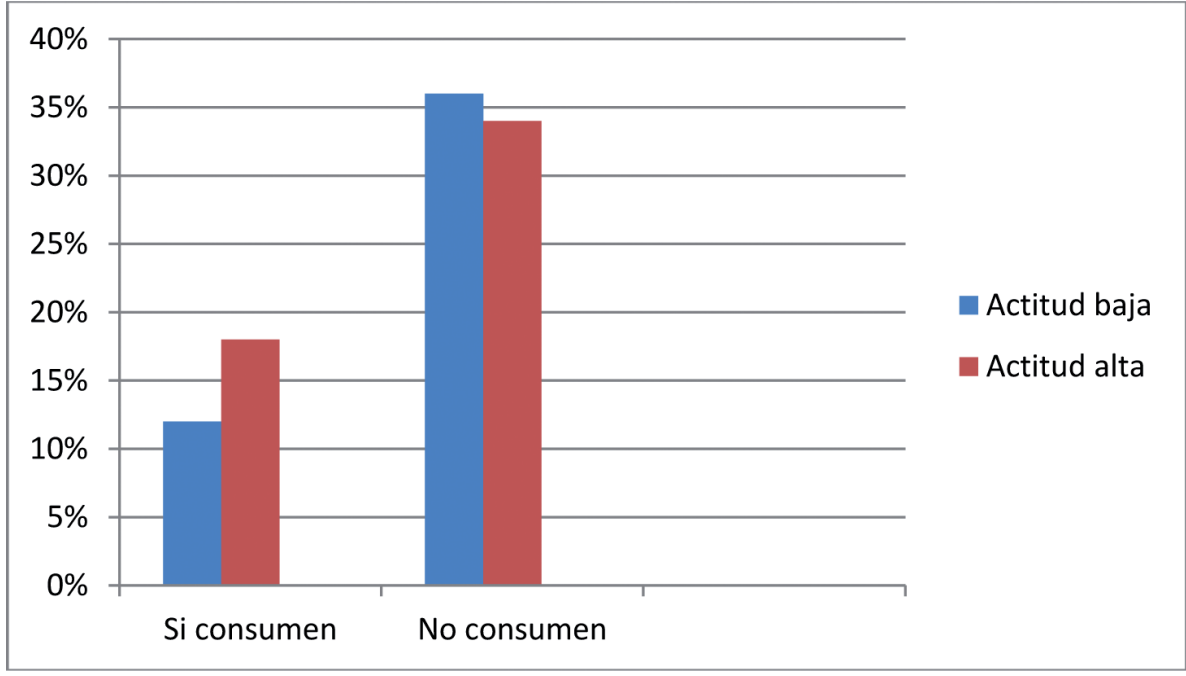

Figura 9. Consumo de droga en prisión

Parece que los sujetos que consumen droga en prisión muestran mejor actitud que los sujetos que no consumen, sin embargo, la relación entre el consumo de drogas en prisión y la actitud mantenida hacia la reeducación es estadísticamente no significativa $\left(\chi^{2}=, 459\right.$; $p>0,05)$.

En el ámbito, contactos con la marginalidad, manifiestan actitud alta los sujetos que no han estado ingresados en Centros de Menores, que han cometido delitos contra salud pública o contra la propiedad, que usaron violencia en la comisión del delito y que consumían drogas durante el tiempo que pasaron en prisión. 
Las actitudes de los jóvenes internados en la prisión de Córdoba ante los procesos de reeducación

María Milagrosa luque Jiménez y María del Rosario Ruiz-Olivares

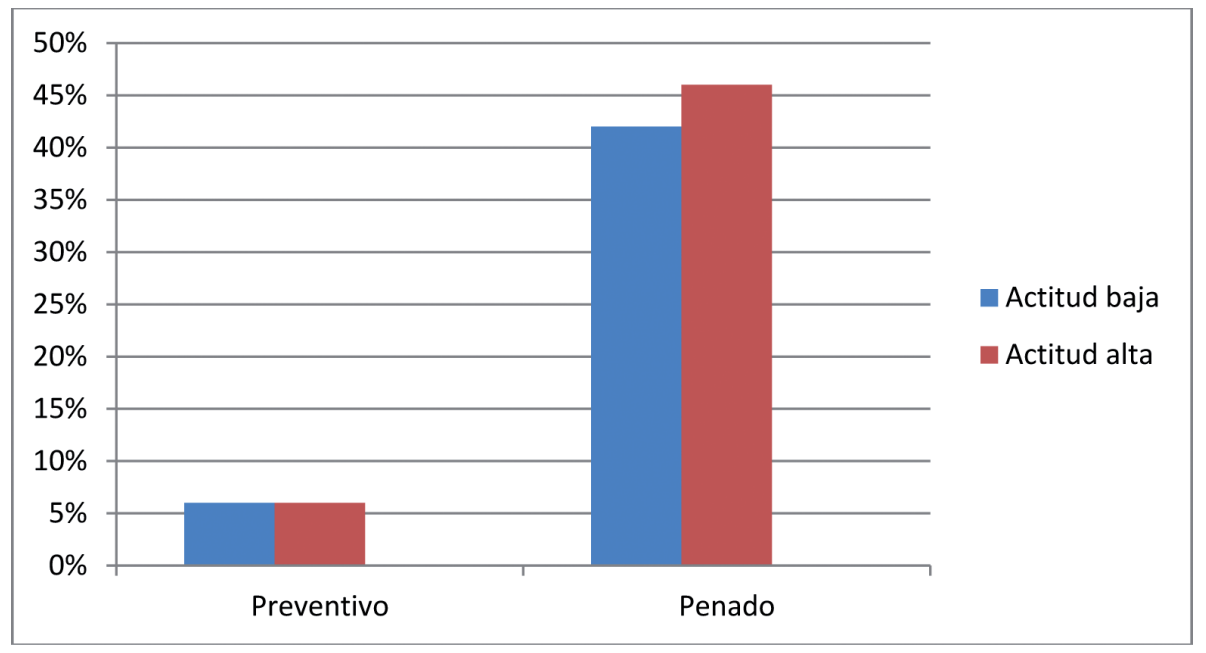

Figura 10. Situación penal

Parece que los sujetos penados tienen mejor actitud que los sujetos preventivos, sin embargo, la relación entre la situación penal y la actitud hacia la reeducación es estadísticamente no significativa $\left(\chi^{2}=, 917 ; p>\right.$ $0,05)$.

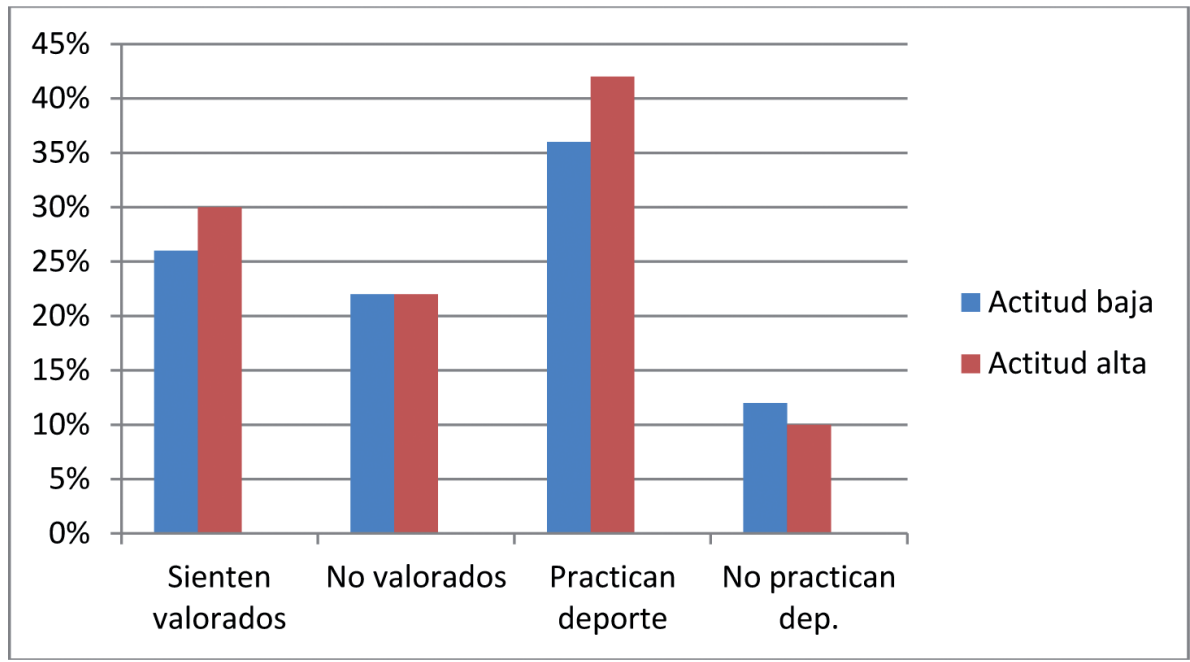

Figura 11. Sentirse valorado por la actividad que realiza en prisión y practicar deporte 
Parece que lo sujetos que se sienten valorados y recompensados por la actividad que realiza en prisión tienen mejor actitud que los sujetos que no se sienten valorados y recompensados, sin embargo, la relación entre el sentirse valorado y recompensado por la actividad realizada en prisión y la actitud mantenida hacia la reeducación es estadísticamente no significativa $\left(\chi^{2}=, 802 ; p>0,05\right)$. Asimismo, los sujetos que practican deporte tienen mejor actitud que los sujetos que no practican, sin embargo, la relación entre la práctica de deporte y la actitud mantenida hacia la reeducación es estadísticamente no significativa $\left(\chi^{2}=, 623 ; p>0,05\right)$.

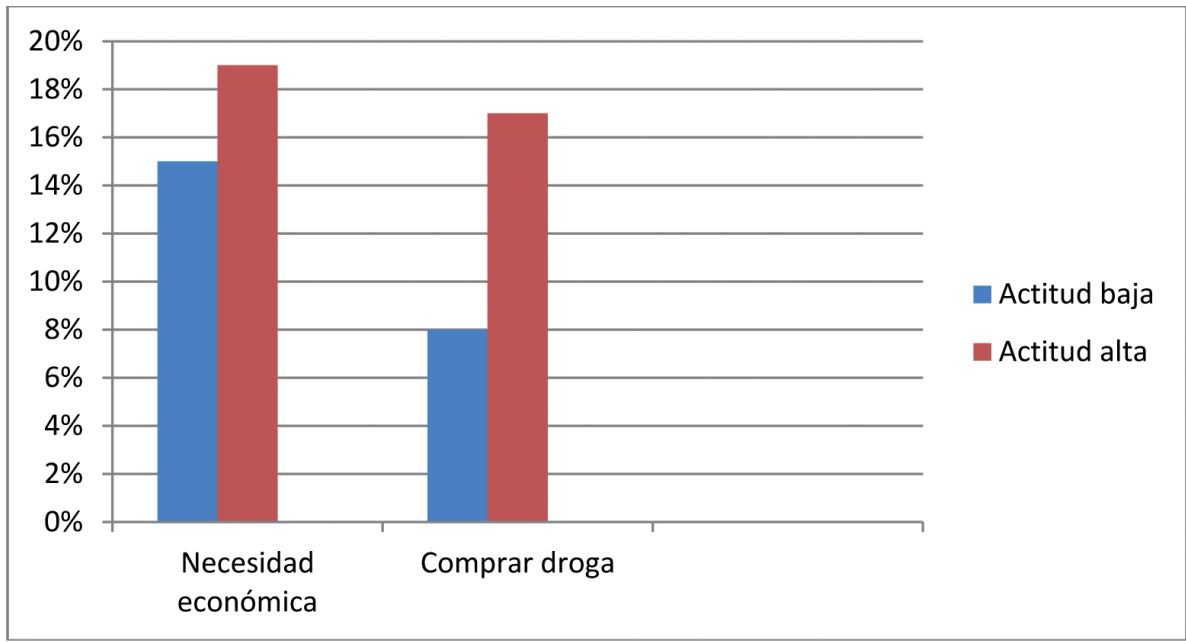

Figura 12. Razones por las que cometió el primer delito

Parece que los sujetos que cometieron su primer delito por necesidad económica o para comprar droga tienen mejor actitud hacia la reeducación que los sujetos que cometieron su delito por otros motivos, sin embargo, la relación entre estas variables y la actitud mantenida hacia la reeducación es estadísticamente no significativa $\left(\chi^{2}=, 877 ; p>0,05\right)$, $\left(\chi^{2}=, 213 ; p>0,05\right)$.

En el ámbito, vida en prisión, tienen actitud alta hacia la reeducación, los sujetos penados, los que practican deporte, los que se sienten valorados y recompensados por la actividad que realiza en prisión, y que cometieron el primer delito por necesidad económica o para comprar droga. 
Las actitudes de los jóvenes internados en la prisión de Córdoba ante los procesos de reeducación

María Milagrosa luque Jiménez y María del Rosario Ruiz-Olivares

\section{Discusión y conclusiones}

Teniendo en cuenta que el objetivo principal de nuestro trabajo de investigación era conocer las actitudes de los internados de la prisión de Córdoba (España), menores de veinticinco año, hacia la reeducación, podemos decir que parece que algo más de la mitad de estos internos tienen una actitud positiva hacia la función reeducadora, de acuerdo a los resultados obtenidos con el cuestionario de Actitudes hacia la Reeducación (C.A.R.). En cualquier caso, tratamos de indagar qué variables socioeducativas y sociodemográficas estaban asociadas a las actitudes. Esta información puede llegar a ser de utilidad a la hora de mejorar la predisposición de los internos para reinsertarse en la sociedad.

Respecto el ámbito, nivel de estudios, se parte de la hipótesis en la que se consideraba el nivel de estudios como un agente que podía influir positivamente en la actitud mantenida. Sin embargo, se observa que el nivel de estudios y la mejora de los estudios en prisión no propician una actitud alta hacia la reeducación. Por otra parte, el recuerdo de la infancia escolar también parece fundamental a la hora de que se produzca un avance hacia la actitud alta. Sin embargo, no se aprecia este dato, ya que los internos que tienen mejor recuerdo escolar manifiestan una actitud baja.

Al igual que ocurre en el trabajo de investigación de Martín Solbes (2006), el nivel de estudios, la mejora de estos estudios en prisión y el recuerdo escolar no propician una actitud alta hacia la reeducación.

En el ámbito de estudios, relaciones familiares, se observa que aunque no tienen relación las variables, muestran actitud alta hacia la reeducación los sujetos que no tienen antecedentes familiares en prisión, han sido bien tratados por la familia, reciben apoyo familiar y los que tienen padres sin graduado escolar.

Estos resultados coinciden con otros trabajos de investigación (Martín, 2006), los internos que muestran una actitud alta hacia la reeducación son aquellos que no tienen antecedentes familiares en prisión, han recibido un trato regular por parte de sus familias y tienen padres con estudios superiores de bachiller.

La familia es un referente muy importante en el niño o adolescente que se forma, por lo que la prevención en el ámbito familiar resulta imprescindible y complementaria a la del ámbito escolar. La familia es el primer grupo de referencia que merece ser objetivo de la prevención de la futura delincuencia (Martín, 2006). 
Se observa que en el tercer ámbito de estudio, los contactos con la marginalidad, aunque las variables tienen poca relación, manifiestan actitud alta los sujetos que no han estado ingresados en centros de menores, que han cometido delitos contra salud pública o contra la propiedad, que usaron violencia en la comisión del delito y que consumían drogas, tanto con anterioridad al ingreso en prisión, como durante el tiempo que pasaron en prisión.

En los resultados de Martín (2006) se puede observar, que en este tercer ámbito de estudio, manifiestan actitud alta los sujetos que no han estado ingresados en centros de menores, que no usaron violencia en la comisión del delito y que consumían drogas tanto, con anterioridad al ingreso en prisión como durante el tiempo que pasaron en prisión.

El problema de la drogadicción es un problema generalizado entre los jóvenes de nuestra sociedad. Son muchos los jóvenes que, de un modo u otro, tienen contactos con el mundo de las drogas, suponiendo estos contactos una situación grave que empora cuando conducen a los jóvenes a prisión. El consumo de drogas, por sí mismo, no produce el ingreso en prisión, aunque de alguna manera relaciona al individuo con ambientes marginales, que guían a los jóvenes por sendas relacionadas de uno u otro modo con la delincuencia. Las razones por las que estas personas consumen drogas son variadas aunque en casi todos los casos, tienen como denominador común, aunque no sea la única causa, la infelicidad y la falta de expectativas de futuro. Indudablemente el componente educativo tiene mucho que decir ante estas situaciones, ya que a través de la óptica pedagógica, los jóvenes pueden descubrir situaciones y experiencias que cambien sus expectativas de vida. Por otro lado, la Institución Penitenciaria debe animar a los jóvenes a participar en programas de desintoxicación, ya que, por un lado, ayudan a abandonar el consumo de drogas, y por otro lado, evita los deterioros personales y sociales propios de las situaciones de drogadicción. Además, si conseguimos disminuir los consumos, es posible disminuir la reincidencia delictiva (Martín, 2006).

Por último, en el cuarto ámbito de estudio, la vida en prisión, se observa que aunque no haya relación entre las variables, los sujetos penados tienen actitud alta hacia la reeducación. Al igual, tanto los sujetos primarios, que ingresan en prisión por primera vez, como los reincidentes mantienen una actitud alta hacia la reeducación. En lo que se refiere al ámbito formativo en los centros penitenciarios, se observa que mantie- 
Las actitudes de los jóvenes internados en la prisión de Córdoba ante los procesos de reeducación

María Milagrosa luque Jiménez y María del Rosario Ruiz-Olivares

nen actitud alta hacia la reeducación los sujetos que practican deporte. Se echa de menos la presencia de la asistencia a talleres ocupacionales y productivos, donde mantienen actitud alta tanto los que no asisten a este tipo de talleres como los que sí. Los sujetos que se sienten valorados por la actividad que realizan en prisión mantienen una actitud alta hacia la reeducación; recordando la importancia de lo emocional en la vida de todos nosotros, mucho más, en las personas encarceladas. Por último, se señalan como variables que definen a los sujetos con actitud alta, los que delinquen por influencias del barrio, y que cometieron el primer delito por necesidad económica y para comprar droga.

En el trabajo de Martín (2006) también se puede apreciar que los sujetos primarios mantienen actitud alta hacia la reeducación. Al contrario que en nuestro caso, en lo que se refiere al ámbito formativo, observamos que mantienen actitud alta hacia la reeducación los sujetos que participan en talleres ocupacionales, talleres productivos y cursos formativos. Echamos de menos la presencia de la práctica deportiva en los sujetos con actitud alta. Asimismo, los sujetos que se sienten valorados por la actividad que realizan en prisión, mantienen actitud alta hacia la reeducación. Por último, tienen actitud alta los que delinquen por influencias de la sociedad y que cometieron el primer delito para comprar droga.

Algunas de estas variables estudiadas no son controlables desde la Institución Penitenciaria, como pueden ser la situación de primario, cometer el delito por influencias de la sociedad, cometer el primer delito para comprar droga, el uso de violencia en el delito, el consumo de droga anterior a prisión, haber ingresado en centros de menores, tener antecedentes familiares en prisión y el nivel de estudios de los padres. Pero desde las prisiones, si se puede trabajar para que los jóvenes internados en prisión participen en talleres ocupacionales, talleres productivos, cursos formativos, se sientan valorados por la actividad que realizan en prisión y asistan a programas de desintoxicación.

Desde la Institución Penitenciaria, se debe trabajar para conseguir que variables como la formación, el deporte, el trabajo o el consumo de drogas, importantes para la vida de cualquier persona, y por consiguiente, también para la vida de los jóvenes en prisión, adquiera la importancia debida y propicien una actitud adecuada hacia la reeducación.

Este trabajo de investigación, más allá de los resultados obtenidos, pretende ser una plataforma de reflexión acerca de la reeducación en 
la Institución Penitenciaria y las actitudes de los internos, teniendo muy presente que como indican Éscamez y Ortega (1986), las actitudes se aprenden y que en un medio como el que estudiamos, invertir medios y tiempo desde un punto de vista educativo, es rentable, ya que el producto educativo y convivencial resultante puede ser perdurable y eficaz en la sociedad.

El análisis de la realidad sobre la situación actual de la reeducación en el medio penitenciario lleva a planteamientos y retos de futuro a corto y a medio plazo. Así, es necesario poner en práctica modelos de enseñanzas de habilidades sociales que respondan a los gustos, expectativas y necesidades de los internos e internas, y a los condicionamientos propios de un medio institucional que se define por la privación de libertad y por su aislamiento social.

Asimismo, es necesario otorgar al trabajo educativo en las prisiones, una útil y eficaz dimensión postpenitenciaria, ya que la razón última de las ofertas culturales y educativas del centro penitenciario, no es la utilización de la cultura o de la educación como coartada para tener a los internos e internas ocupados, garantizando así un mejor orden regimental. Se trata de aprovechar, en la medida de lo posible y a pesar de las dificultades del propio medio, las posibilidades educativas del centro penitenciario y de la comunidad, con vistas a conseguir desarrollar programas específicos de preparación a la libertad orientados a la recuperación de las habilidades y destrezas más básicas, para su nueva convivencia familiar, social y laboral.

"Abogamos por un trabajo tratamental donde primen la normalización social, lo formativo, lo laboral, las relaciones familiares, lo relacional, lo educativo, para convertir la institución penitenciaria en una institución educativa y no traumática" (Martín, 2006, 406).

En este sentido, es interesante hacer hincapié en la necesidad de seguir estudiando la relación entre variables socioeducativas o de otra índole con la actitud que se tiene en un centro penitenciario hacia la reeducación. Así, otras líneas de investigación que surgen a partir de este trabajo de investigación son por ejemplo: conocer las actitudes hacia la reeducación de los jóvenes internados en centros de menores, comparar estas actitudes hacia la reeducación entre hombres y mujeres, comparar estas actitudes hacia la reeducación entre internos españoles y extran- 
Las actitudes de los jóvenes internados en la prisión de Córdoba ante los procesos de reeducación

María Milagrosa luque Jiménez y María del Rosario Ruiz-Olivares

jeros, comparar estas actitudes hacia la reeducación entre internos con carga familiares y sin ella, estudiar las actitudes hacia la reeducación de jóvenes con escasas habilidades cognitivas y comparar con la actitud de personas sin ninguna anormalidad.

En cuanto a las limitaciones de este trabajo, es necesario hacer referencia, por un lado al tamaño de la muestra, metodológicamente trabajar con un número tan reducido de personas hace que los resultados estadísticos no sean tan concluyentes. Es necesario tener en cuenta también el sesgo de deseabilidad social inherente al uso de autoinformes, aunque se garantizaba su anonimato.

\section{Referencias bibliográficas}

Beristain, A. (1985): El delincuente en la democracia. Buenos Aires: Universidad.

Bolívar, A. (2002): La evaluación de valores y actitudes. Madrid: Anaya.

Constitución Española de 31 de octubre de 1978 (B.O.E. nº. 311, de 29 de diciembre).

Escamez, J. y Ortega, P. (1.986): La enseñanza de las actitudes y valores. Nau Libres. Valencia.

Garrido, V. y Montoro, L. (1.992): La reeducación del delincuente juvenil. Los programas de éxito. Tirant lo Blanch. Valencia.

Kaiser, G. (1.988): Introducción a la criminología. Dykinson. Madrid.

Ley Orgánica 10/1995, de 23 de noviembre, del Código Penal.

Ley Orgánica 5/2000, de 12 de enero, reguladora de la responsabilidad penal de los menores.

Martín Solbes, V.M. (2006): Actitudes de los internados en prisión, menores de veintiún años, ante la función reeducadora del medio penitenciario en el ámbito andaluz. Málaga: Servicio de publicaciones de la Universidad de Málaga.

Martín Solbes, V.M. (2009): Los jóvenes internados en prisiones andaluzas. Sus actitudes ante los procesos de reeducación. Revista Pedagogía Social (Universidad Pablo de Olavide). 16, 149-157.

Morales, J. y Moya, M. (2.007): Psicología social. Eudema. Madrid.

Real Decreto 190/1996, de 9 de febrero, por el que se aprueba el Reglamento Penitenciario. 